\title{
LFA-1/ICAM-1 Interaction as a Therapeutic Target in Dry Eye Disease
}

\author{
Stephen C. Pflugfelder, Michael Stern, Steven Zhang, ${ }^{2}$ and Amir Shojaei ${ }^{2}$
}

\begin{abstract}
Dry eye disease (DED) is a common ocular disorder associated with inflammation of the lacrimal gland and ocular surface. The interaction of the integrin lymphocyte function-associated antigen-1 (LFA-1) with its cognate ligand intercellular adhesion molecule-1 (ICAM-1) is known to have important roles in the interaction of a variety of cells involved in immune responses and inflammation, including those prominent in ocular surface inflammation. Lifitegrast, an LFA-1 antagonist that blocks binding of ICAM-1 to LFA-1, has recently been approved in the United States for the treatment of signs and symptoms of DED. In this review, we evaluate research findings to explore the potential role of LFA-1/ICAM-1 interaction in the pathophysiology of DED, and the evidence supporting LFA-1/ICAM-1 interaction as a rational therapeutic target in DED. The results of our review suggest that LFA-1/ICAM-1 interaction may play important roles in the cell-mediated immune response and inflammation associated with DED, including facilitating the homing of dendritic cells to the lymph nodes, interaction of dendritic cells with $\mathrm{T}$ cells and subsequent $\mathrm{T}$ cell activation/differentiation, migration of activated $\mathrm{CD}^{+} \mathrm{T}$ cells from the lymph nodes to the ocular surface, reactivation of $\mathrm{T}$ cells by resident antigen-presenting cells at the ocular surface, and recruitment and retention of LFA-1-expressing T cells in the conjunctival epithelium. Based on the available evidence, inhibition of LFA-1/ICAM-1 interaction represents a rational targeted approach in treating DED. Notably, inhibition of LFA-1/ICAM-1 binding with lifitegrast offers a novel approach to reducing ocular surface inflammation in this condition.
\end{abstract}

Keywords: dry eye disease, ICAM-1, inflammation, LFA-1 antagonist, LFA-1, lifitegrast

\section{Introduction}

D RY EYE DISEASE (DED) is a common ocular disorder affecting the tears and ocular surface ${ }^{1}$ that results in a range of symptoms, including eye pain, dryness, and visual disturbances. Studies have shown that DED exerts a substantial negative impact on the quality of life, function, and work productivity of those affected. ${ }^{2}$ Treatment options for DED include artificial tear substitutes, lubricant gels and ointments, topical cyclosporine, corticosteroids, and punctal plugs. However, there remains an unmet need for effective and well-tolerated treatments that alleviate ocular discomfort and address the etiology of ocular surface damage. Recently, lifitegrast ophthalmic solution $5.0 \%$ has received approval from the US food and drug administration (FDA) for the treatment of the signs and symptoms of DED in adult patients. Lifitegrast blocks the binding of the cell surface proteins lymphocyte function-associated antigen-1 (LFA-1) and intercellular adhesion molecule-1 (ICAM-1) thereby reducing inflammation in DED.

Inflammation of the lacrimal glands and ocular surface, and the immune response in general, are important factors in the etiology of DED. LFA-1 and ICAM-1 are known to have a role in the immune response, and in this review, we draw on research findings in this area to explore their potential role in the pathophysiology of DED, and the body of evidence supporting LFA-1/ICAM-1 interaction as a rational therapeutic target in this condition.

\section{Immunological Basis of DED}

Evidence suggests that DED is an antigen-specific autoimmune inflammatory disease. ${ }^{3,4}$ The immunopathogenesis of DED is hypothesized to involve an inflammatory/immune

\footnotetext{
${ }^{1}$ Baylor College of Medicine, Houston, Texas.

${ }^{2}$ Shire, Lexington, Massachusetts.

(C) Stephen C. Pflugfelder, et al., 2017; Published by Mary Ann Liebert, Inc. This Open Access article is distributed under the terms of the Creative Commons Attribution Noncommercial License (http://creativecommons.org/licenses/by-nc/4.0/) which permits any noncommercial use, distribution, and reproduction in any medium, provided the original author(s) and the source are credited.
} 
cycle consisting of afferent and efferent arms. In the afferent arm, when stress to the ocular surface (environmental, endogenous, and/or microbial, in combination with genetic factors) exceeds a certain threshold, it stimulates the production of proinflammatory cytokines (eg, interleukin [IL]-1, IL-6, and tumor necrosis factor- $\alpha$ [TNF- $\alpha]$ ), matrix metalloproteinases, and chemokines. The resulting proinflammatory milieu induces the maturation of antigenpresenting cells (APCs), and in particular, dendritic cells resident in the ocular tissue. ${ }^{3,4}$ The specific initiating autoantigen has not yet been identified, but it is hypothesized that mature APCs bearing self-antigen migrate to regional lymph nodes through afferent lymphatic vessels where they prime naive $\mathrm{T}$ cells, which then differentiate into the $\mathrm{CD} 4^{+} \mathrm{T}$ helper cell subsets, $T_{H} 1$ and $T_{H} 17$. The effector $T_{H} 1$ and $T_{H} 17$ cells migrate through efferent blood vessels to the ocular surface, where they are thought to induce epithelial damage and tear dysfunction via proinflammatory cytokine release. . $^{3,4}$

In accordance with this model, central to the immunopathogenesis of DED are $\mathrm{CD}^{+}{ }^{+} \mathrm{T}$ cells and dendritic cells. Robust evidence of the role of $\mathrm{CD}^{+} \mathrm{T}$ cells in DED is provided by a study in which $\mathrm{CD} 4^{+} \mathrm{T}$ cells isolated from the cervical lymph nodes of mice with desiccating stressinduced DED were transferred to $\mathrm{T}$ cell-deficient nude mice. ${ }^{5}$ This transfer was sufficient to induce dry eye-like disease in the ocular surface of recipient mice, even in the absence of desiccating stress. ${ }^{5}$ In addition, $\mathrm{CD}^{+} \mathrm{T}$ cells have been shown to be present in lacrimal gland biopsy tissue from patients with Sjögren's syndrome (SS) ${ }^{6}$ and in conjunctival biopsy samples from patients with DED. ${ }^{7}$ A reduction in activated lymphocytes in the conjunctiva of patients with SS has also been seen with DED treatment. ${ }^{8}$ $\mathrm{T}_{\mathrm{H}} 1$ cells are well recognized to secrete the proinflammatory cytokines TNF- $\alpha$, interferon- $\gamma$ (IFN- $\gamma$ ), and IL-2, which activate macrophages, ${ }^{9,10}$ and in the case of IFN- $\gamma$, cause goblet cell dysfunction and death. ${ }^{11-14} \mathrm{~T}_{\mathrm{H}} 17$ cells secrete the cytokine IL-17, which stimulates the production of other proinflammatory molecules, recruits neutrophils, and has been shown to promote corneal epithelial barrier disruption. ${ }^{15,16}$ Evidence supporting a fundamental role of dendritic cells in DED is provided by a study demonstrating accumulation of mature CD11 $\mathrm{c}^{+}$cells (dendritic cells) in the draining cervical lymph nodes within $24 \mathrm{~h}$ of desiccating stress-induced DED in mice, and correlation with the formation of autoreactive $\mathrm{CD}^{+}{ }^{+} \mathrm{T}$ cells. ${ }^{17}$ Furthermore, this study provided evidence that resident APCs in the ocular surface tissue are required for secondary activation of $\mathrm{CD}^{+}$ $\mathrm{T}$ cells in the ocular surface, with local depletion of dendritic cells inhibiting the ability of activated $\mathrm{CD}^{+} \mathrm{T}$ cells to accumulate in ocular surface tissues. ${ }^{17}$

\section{Biology of LFA-1 and ICAM-1}

Integrins are cell surface proteins that play important roles in the interaction of a variety of cells, including those of the immune system. Integrins mediate cell-cell and cellextracellular matrix interactions through bidirectional signaling across the plasma membrane. Structurally, integrins are transmembrane heterodimers consisting of 2 noncovalently associated subunits ( $1 \alpha$ - and $1 \beta$-subunit). Each subunit comprises a single transmembrane domain, a large extracellular region, and a generally short cytoplasmic tail. ${ }^{18-20}$

Integrins normally exist in an inactive state, with the extracellular domains in a bent conformation (Fig. 1). Binding of specific proteins to cytoplasmic domains induces conformational changes that straighten the extracellular domains, thus exposing the ligand-binding site (inside-out signaling; Fig. 1). This conformational change allows integrins to bind to extracellular ligands, resulting in transduction of signals from the outside to the inside of the cell (outsidein signaling). Two extended forms exist with intermediate- or
A

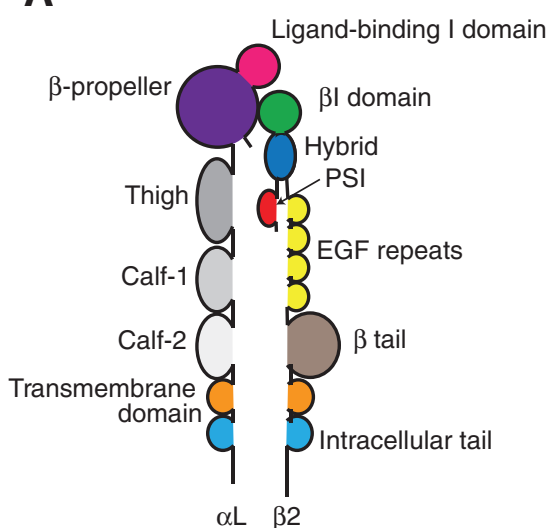

B

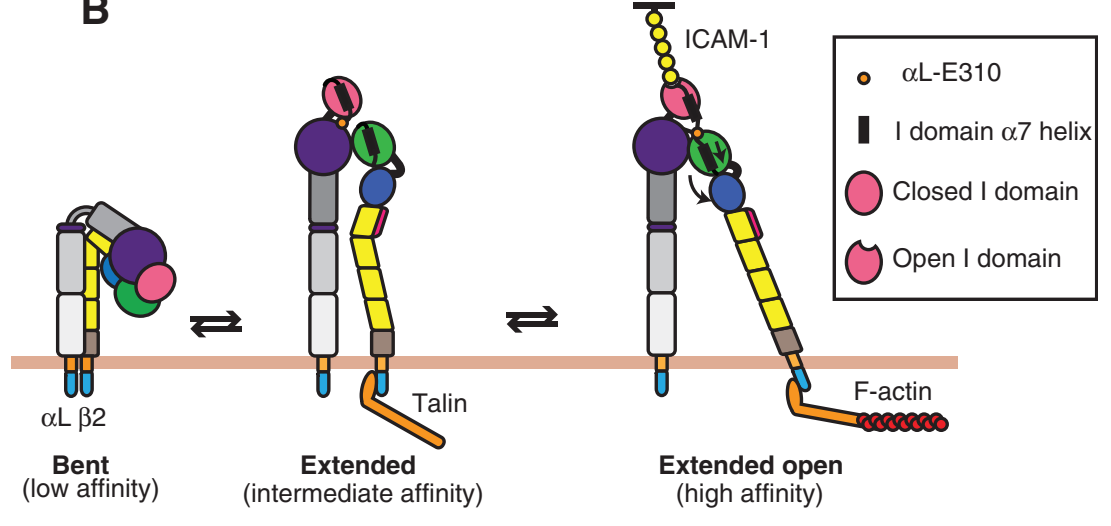

FIG. 1. Schematic representation of the domain structure and conformations of LFA-1. (A) Domain structure of LFA-1. (B) LFA-1 exists in 3 conformations according to binding affinity for ICAM-1. In the resting state, LFA-1 has a low-affinity bent conformation with the cytoplasmic tails in close proximity and the ligand-binding $\alpha \mathrm{L}$ I domain pointing toward the plasma membrane. Inside-out signaling initiated by cytokines, chemokines, or $\mathrm{T}$ cell activation leads to recruitment of Rap1-GTP and then activated talin to the cytoplasmic $\beta 2$ tail of LFA-1, inducing separation of the tails and integrin extension. After extension, the hybrid domain can swing out, pulling down the $\alpha 7$ helix in the $\beta$ I domain. The $\beta$ I domain is then able to bind an internal ligand in the $\alpha \mathrm{L}$ I domain (aL-E310), thus opening the ligand-binding site. Talin interaction with F-actin is important to anchor LFA-1 to the actin cytoskeleton of the cell. Adapted figure reprinted from Front. Immunol., 7, Comrie and Burkhardt, ${ }^{23}$ "Action and Traction: Cytoskeletal Control of Receptor Triggering at the Immunological Synapse," 68, Copyright (2016) with permission. EGF, epidermal growth factor; ICAM-1, intercellular adhesion molecule-1; LFA-1, lymphocyte function-associated antigen-1; PSI, plexin-semaphorin-integrin domain. 
high-affinity binding to the ligand, according to the angle between the hybrid and the $\beta$ I domain (Fig. 1). ${ }^{18,19,21-23}$

LFA- 1 is a member of the $\beta 2$ family of integrins, expressed on the cell surface of leukocytes, and comprising an $\alpha \mathrm{L}$ (CD11a) subunit and a $\beta 2$ (CD18) subunit. ${ }^{19}$ LFA-1 binds with high affinity to ICAM-1, its main ligand, and with lower affinities to ICAM-2 and ICAM-3. ${ }^{24}$ ICAM-1 belongs to the immunoglobulin protein superfamily and contains 5 immunoglobulin-like domains. It is expressed on the cell surface of leukocytes, endothelial cells, keratinocytes, and epithelial cells. ${ }^{25}$

Typical of B-integrins, LFA-1 is generally inactive on circulating leukocytes and requires inside-out signals to activate it so that it can bind to ICAM-1. This process has not been fully elucidated but is thought to be initiated by stimuli received by membrane receptors for cytokines and chemokines, or activation of the $\mathrm{T}$ cell receptor (TCR) by foreign antigens, leading to the activation of phospholipase $\mathrm{C}$ and calcium signaling. Downstream of calcium signaling, the GTPase RAP1 is activated and enables binding of the protein talin-1 to the cytoplasmic 32 tail of LFA-1, in turn enabling a link between LFA-1 and the cytoskeleton through binding of talin to actin. Kindlin-3 also is thought to bind to the 32 -chain, aiding talin binding. Inside-out signaling leads to conformational changes in the extracellular domains of
LFA-1, increasing their affinity for ICAM-1 (Fig. 1). ${ }^{18,19,26,27}$ In addition, several studies show that the distribution of LFA1 into nanoclusters at the cell surface and their coalescence into macroclusters increases the avidity of binding to ICAM-1 in leukocytes. ${ }^{28,29}$ Binding of ICAM-1 to LFA-1 leads to outside-in signaling, with a number of key molecules for this process already assembled at the plasma membrane following inside-out signaling. ${ }^{18}$ Outside-in signaling leads to the transmission of signals from the extracellular domains to the cytoplasm, thus regulating downstream gene expression. ${ }^{26}$

\section{Role of LFA-1/ICAM-1 in the Immunoinflammatory Pathway of DED}

In the following section, we discuss the role that LFA-1/ ICAM-1 interaction plays in the afferent and efferent arms of the immunoinflammatory pathway of DED, represented schematically in Fig. 2.

\section{Role of LFA-1/ICAM-1 in the Afferent Arm of the DED Immunoinflammatory Pathway}

The afferent arm of the immunoinflammatory pathway in DED results in the activation and maturation of dendritic
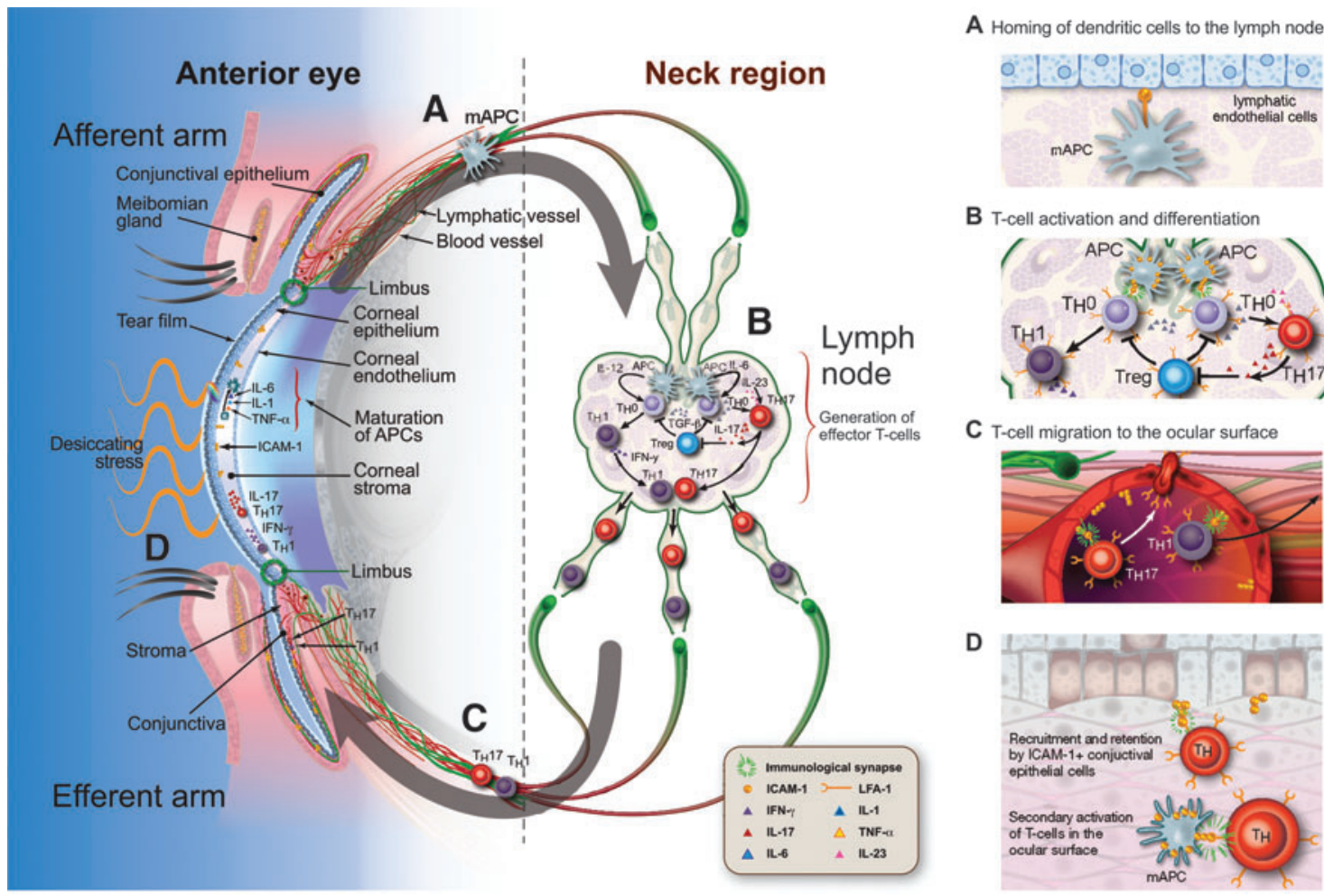

D

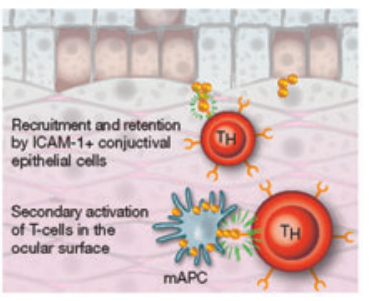

FIG. 2. Potential roles of LFA-1/ICAM-1 interaction in the afferent and efferent arms of the DED immunoinflammatory pathway. Research suggests that LFA-1/ICAM-1 interaction has roles at the indicated points of the inflammatory/immune cycle of DED. These include the homing of dendritic cells to the lymph nodes (A), where their interaction with T cells and subsequent $T$ cell activation and differentiation is facilitated by the formation of the immunological synapse (B). LFA-1/ ICAM-1 interaction also may have roles in the migration of activated CD4 ${ }^{+} \mathrm{T}$ cells from the lymph nodes to the ocular surface (C), reactivation by resident APCs, and recruitment and retention at the conjunctiva (D). Reprinted from Ocular Surface, 14, Perez et al., 36 "Lifitegrast, a Novel Integrin Antagonist for Treatment of Dry Eye Disease," 207-215, Copyright (2016), with permission from Elsevier. APC, antigen-presenting cell; DED, dry eye disease; ICAM-1, intercellular adhesion molecule-1; IFN- $\gamma$, interferon- $\gamma$; IL, interleukin; LFA-1, lymphocyte function-associated antigen-1; mAPC, mature antigen-presenting cell; TGF- $\beta$, transforming growth factor- $\beta$; TNF- $\alpha$, tumor necrosis factor- $\alpha$; Treg, regulatory $\mathrm{T}$ cell. 
cells and their migration to the regional lymph nodes. ${ }^{3,4} \mathrm{~A}$ role for LFA-1/ICAM-1 in this arm of the immunoinflammatory pathway is supported by studies using experimental models of inflammation, in which blockade with antibodies of ICAM-1 on lymphatic endothelial cells or LFA-1 on dendritic cells decreases dendritic cell migration to the lymph nodes. ${ }^{30-32}$ LFA-1 expressed on dendritic cells is generally functionally inactive, with LFA-1-mediated binding to ICAM-1 lost during differentiation from monocyte precursors. ${ }^{28,33}$ However, in vitro exposure of dendritic cells to the chemokine CCL21 under low shear stress, resembling shear stress in the lymphatics, has been shown to activate LFA-1 and restore ICAM-1 binding capacity. ${ }^{33}$ CCL21-CCR7 signaling is necessary for the migration of dendritic cells to the lymph nodes, ${ }^{34}$ and these results support a potential link between LFA-1/ICAM-1 and CCL21 in this process. The authors also extended their study in vivo, demonstrating that the active form of LFA-1 was upregulated in tumor antigen-loaded mature dendritic cells that had migrated into the lymph nodes of patients with melanoma. ${ }^{33}$ Further evidence of the role of LFA-1/ICAM-1 in the migration of dendritic cells to lymph nodes during inflammation is provided by a study, in which imaging techniques were used to demonstrate a strong ICAM-1 presence on the lymphatic endothelial cell surface of inflamed mouse dermal tissue in contact with incoming dendritic cells during their transmigration into lymphatics. ${ }^{35}$ Compared with noninflamed samples, there was a 4-fold increase in the number of adhered dendritic cells surrounded by ICAM-1-rich structures in inflamed samples, which was blocked when dendritic cells were pretreated with an antibody targeting LFA-1. ${ }^{35}$

\section{Role of LFA-1/ICAM-1 in the Efferent Arm of the DED Immunoinflammatory Pathway}

In the efferent arm of the DED immunoinflammatory pathway, naive $\mathrm{T}$ cells are primed in the lymph nodes through interaction with dendritic cells and differentiate to $\mathrm{T}_{\mathrm{H}} 1$ and $\mathrm{T}_{\mathrm{H}} 17$ effector cells. ${ }^{3,4,36}$ These activated $\mathrm{CD}^{+}$ effector $\mathrm{T}$ cells migrate from the lymph nodes to the ocular surface and lacrimal glands, where they exert inflammatory effects. LFA-1 and ICAM-1 may have roles at each step in this pathway, including $\mathrm{T}$ cell activation in the lymph node, $\mathrm{T}$ cell migration, and potentially secondary activation of $\mathrm{T}$ cells at the ocular surface.

\section{T cell activation in the lymph node}

$\mathrm{T}$ cell activation is mediated by the interaction of naive $\mathrm{T}$ cells with dendritic cells and other APCs, and involves the TCR-mediated recognition of antigenic peptide complexed with major histocompatibility complex (MHC) molecules on the surface of APCs. Costimulation signals between molecules on the membranes of the APC and T cell also are required for effective $\mathrm{T}$ cell activation. LFA-1/ICAM-1 is not considered to provide a conventional $\mathrm{T}$ cell costimulation signal, but rather to act as an enhancer of TCR signaling. ${ }^{37} \mathrm{~T}$ cells and APCs make initial contact through transient low-affinity interactions to mediate $\mathrm{T}$ cell scanning for specific antigens on the cell surface of APCs. Several receptor-ligand pairs of adhesion molecules are involved in this process, including LFA-1/ICAM-1, CD2/LFA-3, and LFA-1/ICAM-3. ${ }^{38,39}$ These molecules tether T cell and APC membranes and facilitate TCR-MHC interactions. LFA-1/ ICAM-1 also plays a role in sustaining longer lasting $\mathrm{T}$ cellAPC interactions through the formation of the immunological synapse (Fig. 3). Initially, the immunological synapse consists of an outer ring of TCR-peptide-MHC surrounding a central cluster of LFA-1/ICAM-1 interactions. This later inverts so that the LFA-1/ICAM-1 interactions, termed the peripheral-supramolecular activation cluster, form a ring around the TCR-peptide-MHC cluster. The LFA-1/ICAM-1 interaction may provide stability for the formation of the immunological synapse, allowing it to enhance $\mathrm{T}$ cell sensitivity to the antigen. ${ }^{39,40}$

A number of in vitro studies using human $\mathrm{T}$ cells suggest that the LFA-1/ICAM-1 interaction promotes the differentiation of naive $T$ cells specifically to $T_{H} 1$ cells (relative to $\mathrm{T}_{\mathrm{H}} 2$ ), one of the key effector cell types in DED. In these studies, stimulation of human $\mathrm{T}$ cells through the coreceptor
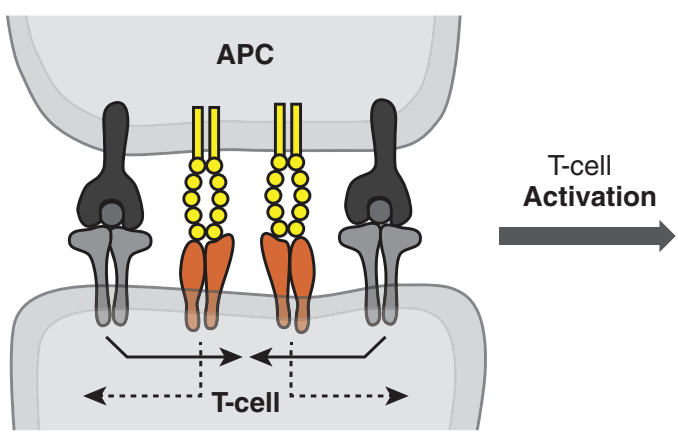

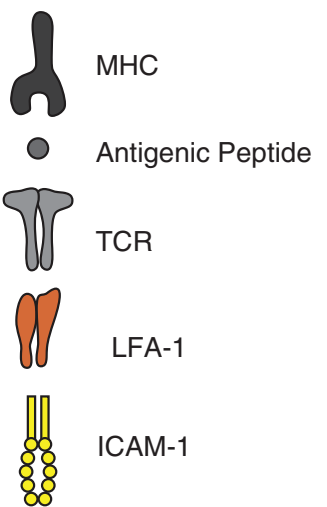

FIG. 3. Role of LFA-1/ICAM-1 interaction in the formation of the immunological synapse. Side view of the formation of an immunological synapse between a T cell and an APC. Left: Early interaction consists of an outer ring of TCR-peptideMHC surrounding a central cluster of LFA-1/ICAM-1 interactions. Right: Signaling molecules then move in the directions shown so that the ring of LFA-1-ICAM-1 surrounds a central cluster of TCR-peptide-MHC. Reprinted from Peptides, 24, Anderson and Siahaan, 57 "Targeting ICAM-1/LFA-1 interaction for controlling autoimmune diseases: designing peptide and small molecule inhibitors," 487-501, Copyright (2003), with permission from Elsevier. APC, antigen-presenting cell; ICAM-1, intercellular adhesion molecule-1; LFA-1, lymphocyte function-associated antigen-1; MHC, major histocompatibility complex; TCR, T cell receptor. 
CD3 plus costimulation of LFA-1 and/or ICAM-1 increased secretion of the $\mathrm{T}_{\mathrm{H}} 1$ cytokines IL- 2 and IFN- $\gamma$, but not the $\mathrm{T}_{\mathrm{H}} 2$ cytokines IL- 4 or IL-5. ${ }^{41-43}$ In another study, nonspecific stimulation of human $\mathrm{T}$ cells with staphylococcal enterotoxin A in combination with ICAM-1 costimulation was shown to induce production of IL- 2 , IFN- $\gamma$, and TNF, but to inhibit production of the $\mathrm{T}_{\mathrm{H}} 2$ cytokine IL-10. ${ }^{44}$ Moreover, IL-2 production was blocked by monoclonal antibodies against ICAM-1 or LFA-1. ${ }^{44}$ These results highlight the importance of LFA-1/ICAM-1 in the $\mathrm{T}_{\mathrm{H}} 1$ immune response, which is generally involved in proinflammatory responses and autoimmune diseases in which cell-mediated immunity dominates.

\section{$T$ cell migration to the ocular surface}

ICAM-1 is important for the migration of lymphocytes from the blood across the vascular endothelium into lymph nodes or inflamed tissues (eg, the ocular surface in DED). During inflammation, ICAM-1 expression is upregulated by proinflammatory cytokines on vascular and lymphatic endothelial cells, as well as other tissue cells, ${ }^{31,45-47}$ and the expression of ICAM-1 has also been shown to be increased in the vascular endothelium of patients with DED. ${ }^{7}$ The process by which lymphocytes adhere to the endothelium during inflammation is termed the leukocyte adhesion cascade. This involves the upregulation of a number of adhesion molecules on the $\mathrm{T}$ cell surface, which bind $\mathrm{E}$ - or P-selectin expressed by the endothelium, thus reducing the speed of the $\mathrm{T}$ cells, which subsequently roll along the vascular wall. ${ }^{48}$ The LFA-1/ICAM-1 interaction plays a role in the firm adhesion of $\mathrm{T}$ cells to the endothelium, followed by transmigration of $\mathrm{T}$ cells across endothelial cells via intracellular signals that lead to cytoskeletal changes necessary for cell movement. ${ }^{48,49}$ Active LFA-1 on T cells signals the remodeling of the F-actin cytoskeleton, which is essential for $\mathrm{T}$ cell adhesion to ICAM-1. 50

\section{$T$ cell activation in the ocular surface}

Once in the ocular surface, there is evidence that CD4 ${ }^{+}$ cells may require secondary activation by resident APCs to maintain effector function. ${ }^{17}$ Given the role of LFA-1/ ICAM-1 in mediating the T cell-dendritic cell interaction, it is reasonable to assume that LFA-1/ICAM-1 could have a role in $\mathrm{T}$ cell activation at the ocular surface, which may further promote the release of proinflammatory cytokines from either the T cells or APCs. However, further research is needed to elucidate the mechanisms and specific cell interactions that are involved.

\section{Recruitment of LFA-1-expressing $T$ cells to the conjunctival epithelium}

The expression of various immunomodulatory molecules has been shown to be increased in the ocular tissues of patients with DED or SS, $, 7,51-54$ and several studies demonstrate upregulation of ICAM-1 expression. ${ }^{7,55,56}$ For example, increased protein expression of ICAM-1 was demonstrated in conjunctival biopsy samples taken from patients with SS or non-SS DED on conjunctival epithelial cells, vascular endothelial cells, and infiltrating lymphocytes in the substantia propria. ${ }^{7}$ In a second study, increased ICAM-1 immunoreactivity was observed in conjunctival epithelial cells and there was a $>3$-fold increase in ICAM-1 gene transcripts in the conjunctival epithelium of patients with SS compared with controls. ${ }^{56}$ A third study also demonstrated increased protein and messenger RNA expression of ICAM-1 in lacrimal and conjunctival epithelial cells from patients with DED. ${ }^{55}$ In this study, ${ }^{55}$ increased ICAM-1 protein expression in lacrimal/conjunctival epithelial cells and LFA-1 protein expression in infiltrating lymphocytes were demonstrated in MRL/lpr mice, which have systemic autoimmune disease that causes SS-like ocular and lacrimal gland inflammation. The authors further investigated the effects of ICAM-1 and LFA-1 inhibition in these mice and found that intraperitoneal administration of monoclonal antibodies targeting ICAM-1 and LFA-1 resulted in a reduction in the number of inflammatory infiltrates in the lacrimal glands. ${ }^{55}$ Notably, the greatest inhibition of cellular infiltration in the conjunctiva was achieved with the administration of a combination of monoclonal antibodies against ICAM-1 and LFA-1, rather than the use of either anti-ICAM-1 or anti-LFA-1 antibody alone. Taken together, these results suggest a potential role of LFA-1/ICAM-1 in the recruitment and retention of LFA-1expressing $\mathrm{T}$ cells to the epithelial cells of the conjunctiva, where they could induce damage via proinflammatory cytokine release.

\section{Targeting LFA-1/ICAM-1 Interaction in DED}

On the basis of the role of LFA-1/ICAM-1 in inflammation and the immune response, several drugs targeting LFA1/ICAM-1 binding have been investigated systemically as anti-inflammatory agents, including efalizumab for psoriasis and lovastatin for rheumatoid arthritis. ${ }^{57}$ Lifitegrast, an LFA-1 antagonist, is the first agent targeting LFA-1/ICAM1 to be approved for the topical treatment of DED. ${ }^{36,58,59}$

Lifitegrast is thought to act as a direct competitive antagonist of the binding of ICAM-1 to LFA-1. ${ }^{36}$ In experiments using a live cell lipid bilayer assay ${ }^{60}$ to mimic the binding of ICAM-1 to LFA-1, lifitegrast inhibited immunologic synapse formation in a dose-dependent manner. ${ }^{61}$ In addition, lifitegrast has been shown to inhibit $\mathrm{T}$ cell attachment to ICAM-1 and cytokine release from stimulated human peripheral blood mononuclear cells. ${ }^{58}$

We speculate that lifitegrast may have potential to act on both the afferent and efferent arms of the immunoinflammatory pathway in DED. Lifitegrast has high aqueous solubility and has demonstrated rapid absorption into the conjunctiva, cornea, humor, and sclera following ophthalmic administration of a single dose in a rat model. ${ }^{62}$ Thus, in the afferent arm, lifitegrast may block LFA-1/ICAM-1 interaction between dendritic cells in the ocular surface and endothelial cells of the lymphatic vessels, thereby inhibiting transendothelial migration and homing of dendritic cells to the draining lymph nodes. In the efferent arm, lifitegrast may inhibit migration of activated $\mathrm{T}$ cells from conjunctival blood vessels into the conjunctiva, their secondary activation in the ocular tissues, and their recruitment and retention in the conjunctival epithelium. It is possible that lifitegrast also has a direct inhibitory effect on cytokine release from T cells. ${ }^{58}$ Following ophthalmic administration of lifitegrast in humans, it is cleared rapidly from the systemic circulation; however, it is transiently present in the plasma (peak plasma concentration in humans is detected within 5 min). ${ }^{59}$ Therefore, in the efferent arm, lifitegrast also may inhibit 
activated $\mathrm{T}$ cells from exiting from the conjunctival blood vessels into the ocular surface tissues. Further studies are needed to test these theories as the exact mechanism and sites of action of lifitegrast have not yet been confirmed.

The clinical efficacy and safety of lifitegrast in DED have been demonstrated across 5 randomized controlled trials, including 1 phase 2 trial $^{63}$ and 4 phase 3 trials: OPUS-1, ${ }^{64}$ OPUS-2, ${ }^{65}$ OPUS-3 (NCT02284516; publication in development), and the long-term, randomized, double-masked safety study SONATA. ${ }^{66}$ In the Phase 2 and OPUS- 1 trials, lifitegrast showed significant improvement from baseline to day 84 versus placebo in the sign endpoint of inferior corneal staining score (Phase 2: secondary endpoint, nominal $P=0.0209$; OPUS-1: coprimary endpoint, $P=0.0007)$. In the OPUS-2 and OPUS-3 trials, lifitegrast-treated participants experienced significantly greater improvement from baseline to day 84 in the symptom endpoint of eye dryness score (visual analogue scale) versus placebo (OPUS-2: coprimary endpoint, $P<0.0001$; OPUS-3: primary endpoint, $P=0.0007)$. In all of the trials, lifitegrast was generally well tolerated. ${ }^{67}$ Following the recent FDA approval of lifitegrast for the signs and symptoms of DED, it will be of interest to further evaluate the utility of the drug in clinical practice and over longer term follow-up.

\section{Conclusions}

The LFA-1/ICAM-1 integrin receptor-ligand pair may play an important role in the cell-mediated immune response and inflammation associated with DED. Research findings support potential functions of the LFA-1/ICAM-1 interaction in both the afferent and efferent arms of the DED immunoinflammatory pathway, and based on the available evidence, inhibition of LFA-1/ICAM-1 interaction represents a rational targeted approach in treating DED. Blocking the binding of LFA-1 to ICAM-1 with lifitegrast shows promise in the treatment of $\mathrm{DED}^{67}$ and offers a novel approach to targeting ocular surface inflammation in this condition.

\section{Acknowledgment}

The authors thank Nasser Malik, PhD, of Excel Scientific Solutions, who provided medical writing assistance funded by SARcode Bioscience, a fully owned company of Shire PLC.

\section{Author Disclosure Statement}

S.P. and M.S. are consultants for Shire PLC. S.Z. and A.S. are employees of Shire PLC and own stock/stock options in Shire PLC.

\section{References}

1. Definition and Classification Subcommittee of the International Dry Eye Workshop. The definition and classification of dry eye disease: report of the definition and classification subcommittee of the international dry eye workShop (2007). Ocul. Surf. 5:75-92, 2007.

2. McDonald, M., Patel, D.A., Keith, M.S., and Snedecor, S.J. Economic and humanistic burden of dry eye disease in Europe, North America, and Asia: a systematic literature review. Ocul. Surf. 14:144-167, 2016.
3. Stern, M.E., Schaumburg, C.S., Dana, R., et al. Autoimmunity at the ocular surface: pathogenesis and regulation. Mucosal Immunol. 3:425-442, 2010.

4. Stern, M.E., Schaumburg, C.S., and Pflugfelder, S.C. Dry eye as a mucosal autoimmune disease. Int. Rev. Immunol. 32:19-41, 2013.

5. Niederkorn, J.Y., Stern, M.E., Pflugfelder, S.C., et al. Desiccating stress induces $\mathrm{T}$ cell-mediated Sjögren's syndrome-like lacrimal keratoconjunctivitis. J. Immunol. 176:3950-3957, 2006.

6. Saito, I., Terauchi, K., Shimuta, M., et al. Expression of cell adhesion molecules in the salivary and lacrimal glands of Sjogren's syndrome. J. Clin. Lab. Anal. 7:180-187, 1993.

7. Stern, M.E., Gao, J., Schwalb, T.A., et al. Conjunctival Tcell subpopulations in Sjögren's and non-Sjögren's patients with dry eye. Invest. Ophthalmol. Vis. Sci. 43:2609-2614, 2002.

8. Kunert, K.S., Tisdale, A.S., Stern, M.E., Smith, J.A., and Gipson, I.K. Analysis of topical cyclosporine treatment of patients with dry eye syndrome: effect on conjunctival lymphocytes. Arch. Ophthalmol. 118:1489-1496, 2000.

9. Mosmann, T.R., and Sad, S. The expanding universe of Tcell subsets: Th1, Th2 and more. Immunol. Today. 17:138146, 1996.

10. Del Prete, G.F., De Carli, M., Mastromauro, C., et al. Purified protein derivative of mycobacterium tuberculosis and excretory-secretory antigen(s) of Toxocara canis expand in vitro human $\mathrm{T}$ cells with stable and opposite (type $1 \mathrm{~T}$ helper or type $2 \mathrm{~T}$ helper) profile of cytokine production. J. Clin. Invest. 88:346-350, 1991.

11. Zhang, X., De Paiva, C.S., Su, Z., et al. Topical interferongamma neutralization prevents conjunctival goblet cell loss in experimental murine dry eye. Exp. Eye Res. 118:117124, 2014.

12. Pflugfelder, S.C., De Paiva, C.S., Moore, Q.L., et al. Aqueous tear deficiency increases conjunctival interferon- $\gamma$ (IFN- $\gamma$ ) expression and goblet cell loss. Invest. Ophthalmol. Vis. Sci. 56:7545-7550, 2015.

13. Coursey, T.G., Tukler Henriksson, J., Barbosa, F.L., de Paiva, C.S., and Pflugfelder, S.C. Interferon-gammainduced unfolded protein response in conjunctival goblet cells as a cause of mucin deficiency in Sjogren syndrome. Am. J. Pathol. 186:1547-1558, 2016.

14. De Paiva, C.S., Villarreal, A.L., Corrales, R.M., et al. Dry eye-induced conjunctival epithelial squamous metaplasia is modulated by interferon-gamma. Invest. Ophthalmol. Vis. Sci. 48:2553-2560, 2007.

15. De Paiva, C.S., Chotikavanich, S., Pangelinan, S.B., et al. IL-17 disrupts corneal barrier following desiccating stress. Mucosal Immunol. 2:243-253, 2009.

16. Chauhan, S.K., El Annan, J., Ecoiffier, T., et al. Autoimmunity in dry eye is due to resistance of Th17 to Treg suppression. J. Immunol. 182:1247-1252, 2009.

17. Schaumburg, C.S., Siemasko, K.F., De Paiva, C.S., et al. Ocular surface APCs are necessary for autoreactive T cellmediated experimental autoimmune lacrimal keratoconjunctivitis. J. Immunol. 187:3653-3662, 2011.

18. Hogg, N., Patzak, I., and Willenbrock, F. The insider's guide to leukocyte integrin signalling and function. Nat. Rev. Immunol. 11:416-426, 2011.

19. Evans, R., Patzak, I., Svensson, L., et al. Integrins in immunity. J. Cell Sci. 122:215-225, 2009.

20. Srichai, M.B., and Zent, R. Integrin structure and function. In: Zent, Z., Pozzi, A., eds. Cell-Extracellular Matrix 
Interactions in Cancer. New York: Springer-Verlag New York; 2010; p. 19-41.

21. Qin, J., Vinogradova, O., and Plow, E.F. Integrin bidirectional signaling: a molecular view. PLoS Biol. 2:e169, 2004.

22. Kinashi, T. Adhere upright: a switchblade-like extension of $\beta 2$ integrins. Immunity. 25:521-522, 2006.

23. Comrie, W.A., and Burkhardt, J.K. Action and traction: cytoskeletal control of receptor triggering at the immunological synapse. Front. Immunol. 7:68, 2016.

24. de Fougerolles, A.R., and Springer, T.A. Intercellular adhesion molecule 3, a third adhesion counter-receptor for lymphocyte function-associated molecule 1 on resting lymphocytes. J. Exp. Med. 175:185-190, 1992.

25. Roebuck, K.A., and Finnegan, A. Regulation of intercellular adhesion molecule-1 (CD54) gene expression. $J$. Leukoc. Biol. 66:876-888, 1999.

26. Verma, N.K., and Kelleher, D. Adaptor regulation of LFA1 signaling in T lymphocyte migration: potential druggable targets for immunotherapies? Eur. J. Immunol. 44:34843499, 2014.

27. Abram, C.L., and Lowell, C.A. The ins and outs of leukocyte integrin signaling. Annu. Rev. Immunol. 27:339362, 2009.

28. Cambi, A., Joosten, B., Koopman, M., et al. Organization of the integrin LFA-1 in nanoclusters regulates its activity. Mol. Biol. Cell. 17:4270-4281, 2006.

29. Borgman, K.J., van Zanten, T.S., Manzo, C., et al. Priming by chemokines restricts lateral mobility of the adhesion receptor LFA-1 and restores adhesion to ICAM-1 nanoaggregates on human mature dendritic cells. PLoS One. 9:e99589, 2014.

30. Rouzaut, A., Garasa, S., Teijeira, A., et al. Dendritic cells adhere to and transmigrate across lymphatic endothelium in response to IFN- $\alpha$. Eur. J. Immunol. 40:3054-3063, 2010.

31. Johnson, L.A., Clasper, S., Holt, A.P., et al. An inflammation-induced mechanism for leukocyte transmigration across lymphatic vessel endothelium. J. Exp. Med. 203:2763-2777, 2006.

32. Nitschké, M., Aebischer, D., Abadier, M., et al. Differential requirement for ROCK in dendritic cell migration within lymphatic capillaries in steady-state and inflammation. Blood. 120:2249-2258, 2012.

33. Eich, C., de Vries, I.J., Linssen, P.C., et al. The lymphoid chemokine CCL21 triggers LFA-1 adhesive properties on human dendritic cells. Immunol. Cell Biol. 89:458-465, 2011.

34. Forster, R., Davalos-Misslitz, A.C., and Rot, A. CCR7 and its ligands: balancing immunity and tolerance. Nat. Rev. Immunol. 8:362-371, 2008.

35. Teijeira, A., Garasa, S., Peláez, R., et al. Lymphatic endothelium forms integrin-engaging 3D structures during DC transit across inflamed lymphatic vessels. J. Invest. Dermatol. 133:2276-2285, 2013.

36. Perez, V.L., Pflugfelder, S.C., Zhang, S., Shojaei, A., and Haque, R. Lifitegrast, a novel integrin antagonist for treatment of dry eye disease. Ocul. Surf. 14:207-215, 2016.

37. Varga, G., Nippe, N., Balkow, S., et al. LFA-1 contributes to signal I of T-cell activation and to the production of $\mathrm{T}_{\mathrm{h}} 1$ cytokines. J. Invest. Dermatol. 130:1005-1012, 2010.

38. de la Fuente, H., Mittelbrunn, M., Sánchez-Martín, L., et al. Synaptic clusters of MHC class II molecules induced on DCs by adhesion molecule-mediated initial T-cell scanning. Mol. Biol. Cell. 16:3314-3322, 2005.
39. Fooksman, D.R., Vardhana, S., Vasiliver-Shamis, G., et al. Functional anatomy of $\mathrm{T}$ cell activation and synapse formation. Annu. Rev. Immunol. 28:79-105, 2010.

40. Davis, D.M., and Dustin, M.L. What is the importance of the immunological synapse? Trends Immunol. 25:323-327, 2004.

41. Jenks, S.A., and Miller, J. Inhibition of IL-4 responses after $T$ cell priming in the context of LFA-1 costimulation is not reversed by restimulation in the presence of CD28 costimulation. J. Immunol. 164:72-78, 2000.

42. Chirathaworn, C., Kohlmeier, J.E., Tibbetts, S.A., et al. Stimulation through intercellular adhesion molecule-1 provides a second signal for $\mathrm{T}$ cell activation. J. Immunol. 168:5530-5537, 2002.

43. Smits, H.H., de Jong, E.C., Schuitemaker, J.H., et al. Intercellular adhesion molecule-1/LFA-1 ligation favors human Th1 development. J. Immunol. 168:1710-1716, 2002.

44. Labuda, T., Wendt, J., Hedlund, G., and Dohlsten, M. ICAM-1 costimulation induces IL-2 but inhibits IL-10 production in superantigen-activated human $\mathrm{CD}^{+} \mathrm{T}$ cells. Immunology. 94:496-502, 1998.

45. Min, J.-K., Kim, Y.-M., Kim, S.W., et al. TNF-related activation-induced cytokine enhances leukocyte adhesiveness: induction of ICAM-1 and VCAM-1 via TNF receptorassociated factor and protein kinase C-dependent NF- $\kappa \mathrm{B}$ activation in endothelial cells. J. Immunol. 175:531-540, 2005.

46. Peebles, R.S., Bochner, B.S., and Schleimer, R.P. Pharmacologic regulation of adhesion molecule function and expression. In: Ruffolo, R.R., Hollinger, M,A,, eds. Inflammation: Mediators and Pathways. Boca Raton: CRC Press; 1995; p. 29-97.

47. Podgrabinska, S., Kamalu, O., Mayer, L., et al. Inflamed lymphatic endothelium suppresses dendritic cell maturation and function via Mac-1/ICAM-1-dependent mechanism. $J$. Immunol. 183:1767-1779, 2009.

48. Hogg, N., Laschinger, M., Giles, K., and McDowall, A. Tcell integrins: more than just sticking points. J. Cell Sci. 116:4695-4705, 2003.

49. Smith, A., Stanley, P., Jones, K., et al. The role of the integrin LFA-1 in T-lymphocyte migration. Immunol. Rev. 218:135-146, 2007.

50. Porter, J.C., Bracke, M., Smith, A., Davies, D., and Hogg, N. Signaling through integrin LFA-1 leads to filamentous actin polymerization and remodeling, resulting in enhanced T cell adhesion. J. Immunol. 168:6330-6335, 2002.

51. Tsubota, K., Fukagawa, K., Fujihara, T., et al. Regulation of human leukocyte antigen expression in human conjunctival epithelium. Invest. Ophthalmol. Vis. Sci. 40:2834, 1999.

52. Brignole, F., Pisella, P.J., Goldschild, M., et al. Flow cytometric analysis of inflammatory markers in conjunctival epithelial cells of patients with dry eyes. Invest. Ophthalmol. Vis. Sci. 41:1356-1363, 2000.

53. Tsubota, K., Fujita, H., Tsuzaka, K., and Takeuchi, T. Quantitative analysis of lacrimal gland function, apoptotic figures, Fas and Fas ligand expression of lacrimal glands in dry eye patients. Exp. Eye Res. 76:233-240, 2003.

54. Gulati, A., Sacchetti, M., Bonini, S., and Dana, R. Chemokine receptor CCR5 expression in conjunctival epithelium of patients with dry eye syndrome. Arch. Ophthalmol. 124:710-716, 2006.

55. Gao, J., Morgan, G., Tieu, D., et al. ICAM-1 expression predisposes ocular tissues to immune-based inflammation 
in dry eye patients and Sjögrens syndrome-like MRL/lpr mice. Exp. Eye Res. 78:823-835, 2004.

56. Jones, D.T., Monroy, D., Ji, Z., Atherton, S.S., and Pflugfelder, S.C. Sjögren's syndrome: cytokine and Epstein-Barr viral gene expression within the conjunctival epithelium. Invest. Ophthalmol. Vis. Sci. 35:3493-3504, 1994.

57. Anderson, M.E., and Siahaan, T.J. Targeting ICAM-1/ LFA-1 interaction for controlling autoimmune diseases: designing peptide and small molecule inhibitors. Peptides. 24:487-501, 2003.

58. Murphy, C.J., Bentley, E., Miller, P.E., et al. The pharmacologic assessment of a novel lymphocyte functionassociated antigen-1 antagonist (SAR 1118) for the treatment of keratoconjunctivitis sicca in dogs. Invest. Ophthalmol. Vis. Sci. 52:3174-3180, 2011.

59. Semba, C.P., Swearingen, D., Smith, V.L., et al. Safety and pharmacokinetics of a novel lymphocyte functionassociated antigen-1 antagonist ophthalmic solution (SAR 1118) in healthy adults. J. Ocul. Pharmacol. Ther. 27:99104, 2011.

60. Hartman, N.C., Nye, J.A., and Groves, J.T. Cluster size regulates protein sorting in the immunological synapse. Proc. Natl. Acad. Sci. U S A. 106:12729-12734, 2009.

61. Haque, H., Gadek, T.R., and Semba, C.P. MoA of lifitegrast, an investigational ICAM-1 decoy for treatment of dry eye. Presented at: 68th Annual Congress of Japan Clinical Ophthalmology; November 13-16, 2014; Kobe, Japan.

62. Rao, V.R., Prescott, E., Shelke, N.B., et al. Delivery of SAR 1118 to the retina via ophthalmic drops and its effectiveness in a rat streptozotocin (STZ) model of diabetic retinopathy (DR). Invest. Ophthalmol. Vis. Sci. 51:51985204, 2010.

63. Semba, C.P., Torkildsen, G.L., Lonsdale, J.D., et al. A phase 2 randomized, double-masked, placebo-controlled study of a novel integrin antagonist (SAR 1118) for the treatment of dry eye. Am. J. Ophthalmol. 153:1050.e1051e1060.e1051, 2012.

64. Sheppard, J.D., Torkildsen, G.L., Lonsdale, J.D., et al. OPUS-1 Study Group. Lifitegrast ophthalmic solution 5.0\% for treatment of dry eye disease: results of the OPUS-1 phase 3 study. Ophthalmology. 121:475-483, 2014.

65. Tauber, J., Karpecki, P., Latkany, R., et al. OPUS-2 Investigators. Lifitegrast ophthalmic solution $5.0 \%$ versus placebo for treatment of dry eye disease: results of the randomized phase III OPUS-2 study. Ophthalmology. 122: 2423-2431, 2015.

66. Donnenfeld, E.D., Karpecki, P.M., Majmudar, P.A., et al. Safety of lifitegrast ophthalmic solution $5.0 \%$ in patients with dry eye disease: a 1-year, multicenter, randomized, placebo-controlled study. Cornea. 35:741-748, 2016.

67. Holland, E.J., Whitley, W.O., Sall, K., et al. Lifitegrast clinical efficacy for treatment of signs and symptoms of dry eye disease across three randomized controlled trials. Curr. Med. Res. Opin. 1-24, 2016.

Received: July 22, 2016 Accepted: October 10, 2016

Address correspondence to:

Stephen C. Pflugfelder, MD

Professor and James and Margaret Elkins

Chair in Ophthalmology

Baylor College of Medicine Cullen Eye Institute 6565 Fannin NC 505

Houston, TX 77030

E-mail: stevenp@bcm.edu 